Kragujevac Journal of Mathematics

Volume 40(1) (2016), Pages 113-124.

\title{
INEQUALITIES FOR THE POLAR DERIVATIVE OF A POLYNOMIAL WITH RESTRICTED ZEROS
}

\author{
AHMAD ZIREH $^{1}$ AND MAHMOOD BIDKHAM ${ }^{2}$
}

\begin{abstract}
For a polynomial $p(z)$ of degree $n$, we consider an operator $D_{\alpha}$ which map a polynomial $p(z)$ into $D_{\alpha} p(z):=(\alpha-z) p^{\prime}(z)+n p(z)$ with respect to $\alpha$. It was proved by Liman et al. [A. Liman, R. N. Mohapatra and W. M. Shah, Inequalities for the Polar Derivative of a Polynomial, Complex Analysis and Operator Theory, 2010] that if $p(z)$ has no zeros in $|z|<1$ then for all $\alpha, \beta \in \mathbb{C}$ with $|\alpha| \geq 1,|\beta| \leq 1$ and $|z|=1$,

$$
\begin{aligned}
\left|z D_{\alpha} p(z)+n \beta \frac{|\alpha|-1}{2} p(z)\right| \leq & \frac{n}{2}\left\{|| \alpha+\beta \frac{|\alpha|-1}{2}|+| z+\beta \frac{|\alpha|-1}{2} \mid\right] \max _{|z|=1}|p(z)| \\
& \left.-\left[\left|\alpha+\beta \frac{|\alpha|-1}{2}\right|-\left|z+\beta \frac{|\alpha|-1}{2}\right|\right] \min _{|z|=1}|p(z)|\right\} .
\end{aligned}
$$

In this paper we extend above inequality for the polynomials having no zeros in $|z|<1$, except $s$-fold zeros at the origin. Our result generalize certain well-known polynomial inequalities.
\end{abstract}

\section{Introduction and Statement of Results}

According to a well known result as Bernstein's inequality on the derivative of a polynomial $p(z)$ of degree $n$, we have

$$
\max _{|z|=1}\left|p^{\prime}(z)\right| \leq n \max _{|z|=1}|p(z)| \text {. }
$$

The result is best possible and equality holds for a polynomial having all its zeros at the origin (see [13] and [4]). The inequality (1.1) can be sharpened, by considering

Key words and phrases. Polynomial, inequality, maximum modulus, polar derivative, restricted zeros.

2010 Mathematics Subject Classification. Primary: 30A10. Secondary: 30C10, 30D15.

Received: February 20, 2015.

Accepted: December 15, 2015. 
the class of polynomials having no zeros in $|z|<1$. In fact, P. Erdôs conjectured and later Lax [10] proved that if $p(z) \neq 0$ in $|z|<1$, then (1.1) can be replaced by

$$
\max _{|z|=1}\left|p^{\prime}(z)\right| \leq \frac{n}{2} \max _{|z|=1}|p(z)| \text {. }
$$

As a refinement of (1.2), Aziz and Dawood [1] proved that if $p(z)$ is a polynomial of degree $n$ having no zeros in $|z|<1$, then

$$
\max _{|z|=1}\left|p^{\prime}(z)\right| \leq \frac{n}{2}\left\{\max _{|z|=1}|p(z)|-\min _{|z|=1}|p(z)|\right\} .
$$

As an improvement of inequality (1.3) Dewan and Hans [7] proved that if $p(z)$ is a polynomial of degree $n$ having no zeros in $|z|<1$, then for any complex number $\beta$ with $|\beta| \leq 1$ and $|z|=1$,

$$
\begin{aligned}
\left|z p^{\prime}(z)+\frac{n \beta}{2} p(z)\right| \leq \frac{n}{2} & \left\{\left(\left|1+\frac{\beta}{2}\right|+\left|\frac{\beta}{2}\right|\right) \max _{|z|=1}|p(z)|\right. \\
& \left.-\left(\left|1+\frac{\beta}{2}\right|-\left|\frac{\beta}{2}\right|\right) \min _{|z|=1}|p(z)|\right\} .
\end{aligned}
$$

Let $\alpha$ be a complex number. For a polynomial $p(z)$ of degree $n, D_{\alpha} p(z)$, the polar derivative of $p(z)$ is defined as

$$
D_{\alpha} p(z)=n p(z)+(\alpha-z) p^{\prime}(z) .
$$

It is easy to see that $D_{\alpha} p(z)$ is a polynomial of degree at most $n-1$ and that $D_{\alpha} p(z)$ generalizes the ordinary derivative in the sense that

$$
\lim _{\alpha \rightarrow \infty}\left[\frac{D_{\alpha} p(z)}{\alpha}\right]=p^{\prime}(z)
$$

For the polar derivative $D_{\alpha} p(z)$, Aziz and Shah [2] proved that if $p(z)$ having all its zeros in $|z| \leq 1$, then

$$
\left|D_{\alpha} p(z)\right| \geq n|\alpha||z|^{n-1} \min _{|z|=1}|p(z)|, \quad|z| \geq 1,
$$

and as an extension to inequality (1.3) they proved that if $p(z)$ is a polynomial of degree $n$ having no zeros in $|z|<1$, then for every complex number $\alpha$ with $|\alpha| \geq 1$,

$$
\max _{|z|=1}\left|D_{\alpha} p(z)\right| \leq \frac{n}{2}\left\{(|\alpha|+1) \max _{|z|=1}|p(z)|-(|\alpha|-1) \min _{|z|=1}|p(z)|\right\} .
$$

Recently Dewan et al. [9] generalized the inequality (1.6) to the polynomial of the form $p(z)=a_{0}+\sum_{\nu=t}^{n} a_{\nu} z^{\nu}, 1 \leq t \leq n$, and proved if $p(z)=a_{0}+\sum_{\nu=t}^{n} a_{\nu} z^{\nu}, 1 \leq t \leq n$, is a polynomial of degree $n$ having no zeros in $|z|<k, k \geq 1$ then for $|\alpha| \geq 1$,

$$
\max _{|z|=1}\left|D_{\alpha} p(z)\right| \leq \frac{n}{1+s_{0}}\left\{\left(|\alpha|+s_{0}\right) \max _{|z|=1}|p(z)|-(|\alpha|-1) \min _{|z|=k}|p(z)|\right\},
$$


where

$$
s_{0}=k^{t+1}\left\{\frac{\left(\frac{t}{n}\right) \frac{\left|a_{t}\right|}{\left|a_{0}\right|-m} k^{t-1}+1}{\left(\frac{t}{n}\right) \frac{\left|a_{t}\right|}{\left|a_{0}\right|-m} k^{t+1}+1}\right\},
$$

and $m=\min _{|z|=k}|p(z)|$.

As a generalization of the inequality (1.7), Bidkham et al. [5] proved, if $p(z)=$ $a_{0}+\sum_{\nu=\mu}^{n} a_{\nu} z^{\nu}, 1 \leq \mu \leq n$, is a polynomial of degree $n$ having no zeros in $|z|<k$, $k \geq 1$ then for $0<r \leq R \leq k$ and $|\alpha| \geq R$,

$$
\begin{aligned}
\max _{|z|=R}\left|D_{\alpha} p(z)\right| \leq & \frac{n}{1+s_{0}^{\prime}}\left\{\left(\frac{|\alpha|}{R}+s_{0}^{\prime}\right) \exp \left\{n \int_{r}^{R} A_{t} d t\right\} \max _{|z|=r}|p(z)|\right. \\
& +\left(s_{0}^{\prime}+1-\left(\frac{|\alpha|}{R}+s_{0}^{\prime}\right) \exp \left\{n \int_{r}^{R} A_{t} d t\right\} \min _{|z|=k}|p(z)|\right\},
\end{aligned}
$$

where

$$
\begin{aligned}
& A_{t}=\frac{\left(\frac{\mu}{n}\right) \frac{\left|a_{\mu}\right|}{\left|a_{0}\right|-m} k^{\mu+1} t^{\mu-1}+t^{\mu}}{t^{\mu+1}+k^{\mu+1}+\left(\frac{\mu}{n}\right)\left(\frac{\left|a_{\mu}\right|}{\left|a_{0}\right|-m}\right)\left(k^{\mu+1} t^{\mu}+k^{2 \mu} t\right)}, \\
& s_{0}^{\prime}=\left(\frac{k}{R}\right)^{\mu+1}\left\{\frac{\left(\frac{\mu}{n}\right) \frac{\left|a_{\mu}\right|}{\left|a_{0}\right|-m} R k^{\mu-1}+1}{\left(\frac{\mu}{n}\right) \frac{\left|a_{\mu}\right|}{R\left(\left|a_{0}\right|-m\right)} k^{\mu+1}+1}\right\},
\end{aligned}
$$

and $m=\min _{|z|=k}|p(z)|$.

As an improvement and generalization to the inequalities (1.6) and (1.4), Liman et al. [11] proved that if $p(z)$ is a polynomial of degree $n$ having no zeros in $|z|<1$, then for all $\alpha, \beta$ with $|\alpha| \geq 1,|\beta| \leq 1$ and $|z|=1$,

$$
\begin{aligned}
& \left|z D_{\alpha} p(z)+n \beta \frac{|\alpha|-1}{2} p(z)\right| \leq \frac{n}{2}\left\{\left(\left|\alpha+\beta \frac{|\alpha|-1}{2}\right|+\left|z+\beta \frac{|\alpha|-1}{2}\right|\right) \max _{|z|=1}|p(z)|\right. \\
& \left.-\left(\left|\alpha+\beta \frac{|\alpha|-1}{2}\right|-\left|z+\beta \frac{|\alpha|-1}{2}\right|\right) \min _{|z|=1}|p(z)|\right\} \text {. }
\end{aligned}
$$

In this paper, we first obtain the following generalization of polynomial inequality (1.5), as follows:

Theorem 1.1. Let $p(z)$ be a polynomial of degree $n$, having all its zeros in $|z| \leq 1$, with $s$-fold zeros at the origin, then

$$
\left|z D_{\alpha} p(z)+\beta \frac{(n+s)(|\alpha|-1)}{2} p(z)\right| \geq\left|n \alpha+\beta \frac{(n+s)(|\alpha|-1)}{2}\right||z|^{n} \min _{|z|=1}|p(z)|,
$$

for every real or complex numbers $\beta$, $\alpha$ with $|\beta| \leq 1,|\alpha| \geq 1$ and $|z| \geq 1$. The result is best possible and equality holds for the polynomials $p(z)=a z^{n}$.

If we take $s=0$ in Theorem 1.1, we have 
Corollary 1.1. If $p(z)$ is a polynomial of degree $n$, having all its zeros in $|z| \leq 1$, then for $|\beta| \leq 1,|\alpha| \geq 1$ and $|z| \geq 1$, we have

$$
\left|z D_{\alpha} p(z)+n \beta \frac{|\alpha|-1}{2} p(z)\right| \geq n\left|\alpha+\beta \frac{|\alpha|-1}{2}\right||z|^{n} \min _{|z|=1}|p(z)| .
$$

For $\beta=0$ the inequality (1.10) reduces to inequality (1.5).

Next by using Theorem 1.1, we generalize the inequality (1.8).

Theorem 1.2. Let $p(z)$ be a polynomial of degree $n$ does not vanish in $|z|<1$, except $s$-fold zeros at the origin, then for all $\alpha, \beta \in \mathbb{C}$ with $|\alpha| \geq 1,|\beta| \leq 1$ and $|z|=1$, we have

$$
\begin{aligned}
& \left|z D_{\alpha} p(z)+\beta \frac{(n+s)(|\alpha|-1)}{2} p(z)\right| \\
\leq & \frac{1}{2}\left[\left\{\left|n \alpha+\beta \frac{(n+s)(|\alpha|-1)}{2}\right|+\left|(n-s) z+s \alpha+\beta \frac{(n+s)(|\alpha|-1)}{2}\right|\right\} \max _{|z|=1}|p(z)|\right. \\
& \left.-\left\{\left|n \alpha+\beta \frac{(n+s)(|\alpha|-1)}{2}\right|-\left|(n-s) z+s \alpha+\beta \frac{(n+s)(|\alpha|-1)}{2}\right|\right\} \min _{|z|=1}|p(z)|\right] .
\end{aligned}
$$

If we take $s=0$ in Theorem 1.2, then the inequality (1.11) reduces to the inequality (1.8).

Theorem 1.2 simplifies to the following result by taking $\beta=0$.

Corollary 1.2. Let $p(z)$ be a polynomial of degree $n$ does not vanish in $|z|<1$, except $s$-fold zeros at the origin, then for any $\alpha \in \mathbb{C}$ with $|\alpha| \geq 1$ and $|z|=1$, we have

$$
\left.\left|D_{\alpha} p(z)\right| \leq \frac{1}{2}\{n|\alpha|+|(n-s) z+s \alpha|) \max _{|z|=1}|p(z)|-(n|\alpha|-|(n-s) z+s \alpha|) \min _{|z|=1}|p(z)|\right\} .
$$

Dividing two sides of inequality (1.11) by $|\alpha|$ and letting $|\alpha| \rightarrow \infty$, we have the following generalization of the inequality (1.4).

Corollary 1.3. Let $p(z)$ be a polynomial of degree $n$, having no zeros in $|z|<1$, except $s$-fold zeros at the origin, then for any $\beta \in \mathbb{C}$ with $|\beta| \leq 1$, and $|z|=1$ we have

$$
\begin{aligned}
\left|z p^{\prime}(z)+\frac{\beta(n+s)}{2} p(z)\right| \leq \frac{1}{2}\{ & \left(\left|n+\beta \frac{n+s}{2}\right|+\left|s+\beta \frac{n+s}{2}\right|\right) \max _{|z|=1}|p(z)| \\
& \left.-\left(\left|n+\beta \frac{n+s}{2}\right|-\left|s+\beta \frac{n+s}{2}\right|\right) \min _{|z|=1}|p(z)|\right\} .
\end{aligned}
$$

\section{LEMMAS}

For the proofs of these theorems, we need the following lemmas. The first lemma is due to Laguerre [12].

Lemma 2.1. If all the zeros of an $n^{\text {th }}$ degree polynomial $p(z)$ lie in a circular region $C$ and $w$ is any zero of $D_{\alpha} p(z)$, then at most one of the points $w$ and $\alpha$ may lie outside $C$. 
Lemma 2.2. Let $p(z)$ is a polynomial of degree $n$, has no zero in $|z|<1$, then on $|z|=1$,

$$
\left|p^{\prime}(z)\right| \leq\left|q^{\prime}(z)\right|
$$

where $q(z)=z^{n} \overline{p(1 / \bar{z})}$.

The above lemma is due to Chan and Malik [6].

Lemma 2.3. If $p(z)$ is a polynomial of degree $n$, having all its zeros in the closed disk $|z| \leq 1$, then on $|z|=1$,

$$
\left|q^{\prime}(z)\right| \leq\left|p^{\prime}(z)\right|
$$

where $q(z)=z^{n} \overline{p(1 / \bar{z})}$.

Proof. Since $p(z)$ has all its zeros in $|z| \leq 1$, therefore $q(z)$ has no zero in $|z|<1$. Now applying Lemma 2.2 to the polynomial $q(z)$ and the result follows.

The following lemma is due to Aziz and Shah [3].

Lemma 2.4. If $p(z)$ is a polynomial of degree $n$, having all its zeros in the closed disk $|z| \leq 1$, with $s$-fold zeros at the origin, then

$$
\left|p^{\prime}(z)\right| \geq \frac{n+s}{2}|p(z)|, \quad|z|=1 .
$$

Lemma 2.5. If $p(z)$ is a polynomial of degree $n$, having all its zeros in the closed disk $|z| \leq 1$, with $s$-fold zeros at the origin, then for all real or complex number $\alpha$ with $|\alpha| \geq 1$ and $|z|=1$, we have

$$
\left|D_{\alpha} p(z)\right| \geq \frac{(n+s)(|\alpha|-1)}{2}|p(z)| .
$$

The above lemma is due to K. K. Dewan and A. Mir [8].

Lemma 2.6. If $p(z)$ is a polynomial of degree $n$ with $s$-fold zeros at the origin, then for all $\alpha, \beta \in \mathbb{C}$ with $|\beta| \leq 1,|\alpha| \geq 1$ and $|z|=1$, we have

$$
\left|z D_{\alpha} p(z)+\beta \frac{(n+s)(|\alpha|-1)}{2} p(z)\right| \leq\left|n \alpha+\beta \frac{(n+s)(|\alpha|-1)}{2}\right| \max _{|z|=1}|p(z)| .
$$

Proof. Let $M=\max _{|z|=1}|p(z)|$, if $|\lambda|<1$, then $|\lambda p(z)|<\left|M z^{n}\right|$ for $|z|=1$. Therefore it follows by Rouche's Theorem that the polynomial $G(z)=M z^{n}-\lambda p(z)$ has all its zeros in $|z|<1$ with $s$-fold zeros at the origin. By using Lemma 2.5, to the polynomial $G(z)$, we have for every real or complex number $\alpha$ with $|\alpha| \geq 1$ and for $|z|=1$,

or

$$
\left|z D_{\alpha} G(z)\right| \geq \frac{(n+s)(|\alpha|-1)}{2}|G(z)|
$$

$$
\left|n \alpha M z^{n}-\lambda z D_{\alpha} p(z)\right| \geq \frac{(n+s)(|\alpha|-1)}{2}\left|M z^{n}-\lambda p(z)\right| .
$$


On the other hand by Lemma 2.1 all the zeros of $D_{\alpha} G(z)=n \alpha M z^{n-1}-\lambda D_{\alpha} p(z)$ lie in $|z|<1$, where $|\alpha| \geq 1$. Therefore for any $\beta$ with $|\beta| \leq 1$, Rouche's Theorem implies that all the zeros of

$$
n \alpha M z^{n}-\lambda z D_{\alpha} p(z)+\beta \frac{(n+s)(|\alpha|-1)}{2}\left(M z^{n}-\lambda p(z)\right),
$$

lie in $|z|<1$. This conclude that the polynomial

$$
T(z)=\left(n \alpha+\beta \frac{(n+s)(|\alpha|-1)}{2}\right) M z^{n}-\lambda\left(z D_{\alpha} p(z)+\beta \frac{(n+s)(|\alpha|-1)}{2} p(z)\right),
$$

will have no zeros in $|z| \geq 1$. This implies that for every $\beta$ with $|\beta|<1$ and $|z|=1$,

$$
\left|z D_{\alpha} p(z)+\beta \frac{(n+s)(|\alpha|-1)}{2} p(z)\right| \leq\left|n \alpha+\beta \frac{(n+s)(|\alpha|-1)}{2}\right| M .
$$

If the inequality (2.3) is not true, then there is a point $z=z_{0}$ with $\left|z_{0}\right| \geq 1$, such that

$$
\left|n \alpha+\beta \frac{(n+s)(|\alpha|-1)}{2}\right| M<\left|z_{0} D_{\alpha} p\left(z_{0}\right)+\beta \frac{(n+s)(|\alpha|-1)}{2} p\left(z_{0}\right)\right| .
$$

Take

$$
\lambda=\frac{\left(n \alpha+\beta \frac{(n+s)(|\alpha|-1)}{2}\right) M}{z_{0} D_{\alpha} p\left(z_{0}\right)+\beta \frac{(n+s)(|\alpha|-1)}{2} p\left(z_{0}\right)},
$$

then $|\lambda|<1$ and with this choice of $\lambda$, we have $T\left(z_{0}\right)=0$ for $\left|z_{0}\right| \geq 1$, from (2.2). But this contradicts the fact that $T(z) \neq 0$ for $|z| \geq 1$. For $\beta$ with $|\beta|=1$, inequality (2.3) follows by continuity. This completes the proof of Lemma 2.6.

Lemma 2.7. If $p(z)$ is a polynomial of degree $n$ with $s$-fold zeros at the origin, then for all $\alpha, \beta \in \mathbb{C}$ with $|\beta| \leq 1,|\alpha| \geq 1$ and $|z|=1$, we have

$$
\begin{aligned}
& \left|z D_{\alpha} p(z)+\beta \frac{(n+s)(|\alpha|-1)}{2} p(z)\right|+\left|z D_{\alpha} Q(z)+\beta \frac{(n+s)(|\alpha|-1)}{2} Q(z)\right| \\
\leq & \left\{\left|n \alpha+\beta \frac{(n+s)(|\alpha|-1)}{2}\right|+\left|(n-s) z+s \alpha+\beta \frac{(n+s)(|\alpha|-1)}{2}\right|\right\} \max _{|z|=1}|p(z)|,
\end{aligned}
$$

where $Q(z)=z^{n+s} \overline{p(1 / \bar{z})}$.

Proof. Let $M=\max _{|z|=1}|p(z)|$. For $\lambda$ with $|\lambda|>1$, it follows by Rouche's Theorem that the polynomial $G(z)=p(z)-\lambda M z^{s}$ has no zeros in $|z|<1$, except $s$-fold zeros at the origin. Consequently the polynomial

$$
H(z)=z^{n+s} \overline{G(1 / \bar{z})},
$$

has all its zeros in $|z| \leq 1$ with $s$-fold zeros at the origin, also $|G(z)|=|H(z)|$ for $|z|=1$. Since all the zeros of $H(z)$ lie in $|z| \leq 1$, therefore, for $\delta$ with $|\delta|>1$, by 
Rouche's Theorem all the zeros of $G(z)+\delta H(z)$ lie in $|z| \leq 1$. Hence by Lemma 2.5 for every $\alpha$ with $|\alpha| \geq 1$, and $|z|=1$, we have

$$
\frac{(n+s)(|\alpha|-1)}{2}|G(z)+\delta H(z)| \leq\left|z D_{\alpha}(G(z)+\delta H(z))\right| \text {. }
$$

Now using a similar argument as used in the proof of Lemma 2.6, we get for every real or complex number $\beta$ with $|\beta| \leq 1$ and $|z| \geq 1$,

$$
\left|z D_{\alpha} G(z)+\beta \frac{(n+s)(|\alpha|-1)}{2} G(z)\right| \leq\left|z D_{\alpha} H(z)+\beta \frac{(n+s)(|\alpha|-1)}{2} H(z)\right| .
$$

Therefore by the equalities

$$
H(z)=z^{n+s} \overline{G(1 / \bar{z})}=z^{n+s} \overline{p(1 / \bar{z})}-\bar{\lambda} M z^{n}=Q(z)-\bar{\lambda} M z^{n}
$$

or

$$
H(z)=Q(z)-\bar{\lambda} M z^{n}
$$

and substitute for $G(z)$ and $H(z)$ in (2.4) we get

$$
\begin{aligned}
& \left|\left(z D_{\alpha} p(z)+\beta \frac{(n+s)(|\alpha|-1)}{2} p(z)\right)-\lambda\left((n-s) z+s \alpha+\beta \frac{(n+s)(|\alpha|-1)}{2}\right) M z^{s}\right| \\
\leq & \left|\left(z D_{\alpha} Q(z)+\beta \frac{(n+s)(|\alpha|-1)}{2} Q(z)\right)-\bar{\lambda}\left(n \alpha+\beta \frac{(n+s)(|\alpha|-1)}{2}\right) M z^{n}\right| .
\end{aligned}
$$

This implies

$$
\begin{aligned}
& \left|z D_{\alpha} p(z)+\beta \frac{(n+s)(|\alpha|-1)}{2} p(z)\right|-\left|(n-s) z+s \alpha+\beta \frac{(n+s)(|\alpha|-1)}{2}\right|\left|\lambda M z^{s}\right| \\
& 2.5) \leq\left|\left(z D_{\alpha} Q(z)+\beta \frac{(n+s)(|\alpha|-1)}{2} Q(z)\right)-\bar{\lambda}\left(n \alpha+\beta \frac{(n+s)(|\alpha|-1)}{2}\right) M z^{n}\right| .
\end{aligned}
$$

As $|p(z)|=|Q(z)|$ for $|z|=1$, i.e., $\max _{|z|=1}|p(z)|=\max _{|z|=1}|Q(z)|=M$, by using Lemma 2.6 for $Q(z)$, we obtain for $|z|=1$,

$$
\left|z D_{\alpha} Q(z)+\beta \frac{(n+s)(|\alpha|-1)}{2} Q(z)\right|<|\lambda|\left|n \alpha+\beta \frac{(n+s)(|\alpha|-1)}{2}\right| M .
$$

Thus taking suitable choice of argument of $\lambda$, result is

$$
\begin{aligned}
& \left|\left(z D_{\alpha} Q(z)+\beta \frac{(n+s)(|\alpha|-1)}{2} Q(z)\right)-\bar{\lambda}\left(n \alpha+\beta \frac{(n+s)(|\alpha|-1)}{2}\right) M z^{n}\right| \\
(2.6)= & |\lambda|\left|n \alpha+\beta \frac{(n+s)(|\alpha|-1)}{2}\right| M-\left|z D_{\alpha} Q(z)+\beta \frac{(n+s)(|\alpha|-1)}{2} Q(z)\right| .
\end{aligned}
$$

By combining right hand side of (2.5) and (2.6) we get for $|z|=1$ and $|\beta| \leq 1$,

$$
\begin{aligned}
& \left|z D_{\alpha} p(z)+\beta \frac{(n+s)(|\alpha|-1)}{2} p(z)\right|-|\lambda|\left|(n-s) z+s \alpha+\beta \frac{(n+s)(|\alpha|-1)}{2}\right| M \\
\leq & |\lambda|\left|n \alpha+\beta \frac{(n+s)(|\alpha|-1)}{2}\right| M-\left|z D_{\alpha} Q(z)+\beta \frac{(n+s)(|\alpha|-1)}{2} Q(z)\right|,
\end{aligned}
$$


i.e.,

$$
\begin{aligned}
& \left|z D_{\alpha} p(z)+\beta \frac{(n+s)(|\alpha|-1)}{2} p(z)\right|+\left|z D_{\alpha} Q(z)+\beta \frac{(n+s)(|\alpha|-1)}{2} Q(z)\right| \\
\leq & |\lambda|\left\{\left|n \alpha+\beta \frac{(n+s)(|\alpha|-1)}{2}\right|+\left|(n-s) z+s \alpha+\beta \frac{(n+s)(|\alpha|-1)}{2}\right|\right\} M .
\end{aligned}
$$

Taking $|\lambda| \rightarrow 1$, we have

$$
\begin{aligned}
& \left|z D_{\alpha} p(z)+\beta \frac{(n+s)(|\alpha|-1)}{2} p(z)\right|+\left|z D_{\alpha} Q(z)+\beta \frac{(n+s)(|\alpha|-1)}{2} Q(z)\right| \\
\leq & \left\{\left|n \alpha+\beta \frac{(n+s)(|\alpha|-1)}{2}\right|+\left|(n-s) z+s \alpha+\beta \frac{(n+s)(|\alpha|-1)}{2}\right|\right\} M .
\end{aligned}
$$

This gives the result.

The following lemma is due to Zireh [14].

Lemma 2.8. If $p(z)=\sum_{\nu=0}^{n} a_{\nu} z^{\nu}$ is a polynomial of degree $n$, having all its zeros in $|z|<k$, $(k>0)$, then $m<k^{n}\left|a_{n}\right|$, where $m=\min _{|z|=k}|p(z)|$.

\section{Proof of the Theorems}

Proof of Theorem 1.1. If $p(z)$ has a zero on $|z|=1$, then the inequality (1.9) is trivial. Therefore we assume that $p(z)$ has all its zeros in $|z|<1$. Let $m=\min _{|z|=1}|p(z)|$, then $m>0$ and $|p(z)| \geq m$ where $|z|=1$. Therefore, for $|\lambda|<1$, it follows by Rouche's Theorem and Lemma 2.8 that the polynomial $G(z)=p(z)-\lambda m z^{n}$ is of degree $n$ and has all its zeros in $|z|<1$ with $s$-fold zeros at the origin. By using Lemma 2.1, $D_{\alpha} G(z)=D_{\alpha} p(z)-\alpha \lambda m n z^{n-1}$, has all its zeros in $|z|<1$, where $|\alpha| \geq 1$. Applying Lemma 2.5 to the polynomial $G(z)$, yields

$$
\left|z D_{\alpha} G(z)\right| \geq \frac{(n+s)(|\alpha|-1)}{2}|G(z)|, \quad|z|=1 .
$$

Since $z D_{\alpha} G(z)$ has all its zeros in $|z|<1$, by using Rouche's Theorem, it can be easily verifies from (3.1), that the polynomial

$$
z D_{\alpha} G(z)+\beta \frac{(n+s)(|\alpha|-1)}{2} G(z)
$$

has all its zeros in $|z|<1$, where $|\beta|<1$.

Substituting for $G(z)$, we conclude that the polynomial

$$
T(z)=\left(z D_{\alpha} p(z)+\beta \frac{(n+s)(|\alpha|-1)}{2} p(z)\right)-\lambda m z^{n}\left(n \alpha+\beta \frac{(n+s)(|\alpha|-1)}{2}\right),
$$

will have no zeros in $|z| \geq 1$. This implies for every $\beta$ with $|\beta|<1$ and $|z| \geq 1$,

$$
\left|z D_{\alpha} p(z)+\beta \frac{(n+s)(|\alpha|-1)}{2} p(z)\right| \geq m\left|z^{n}\right|\left|n \alpha+\beta \frac{(n+s)(|\alpha|-1)}{2}\right| .
$$


If the inequality (3.3) is not true, then there is a point $z=z_{0}$ with $\left|z_{0}\right| \geq 1$ such that

$$
\left|z_{0} D_{\alpha} p\left(z_{0}\right)+\beta \frac{(n+s)(|\alpha|-1)}{2} p\left(z_{0}\right)\right|<m\left|z_{0}^{n}\right|\left|n \alpha+\beta \frac{(n+s)(|\alpha|-1)}{2}\right| .
$$

Take

$$
\lambda=\frac{z_{0} D_{\alpha} p\left(z_{0}\right)+\beta \frac{(n+s)(|\alpha|-1)}{2} p\left(z_{0}\right)}{m z_{0}^{n}\left(n \alpha+\beta \frac{(n+s)(|\alpha|-1)}{2}\right)},
$$

then $|\lambda|<1$ and with this choice of $\lambda$, we have $T\left(z_{0}\right)=0$ for $\left|z_{0}\right| \geq 1$, from (3.2). But this contradicts the fact that $T(z) \neq 0$ for $|z| \geq 1$. For $\beta$ with $|\beta|=1$, inequality (3.3) follows by continuity. This completes the proof of Theorem 1.1.

Proof of Theorem 1.2. Under the assumption of Theorem 1.2, we can write $p(z)=$ $z^{s} h(z)$, where the polynomial $h(z) \neq 0$ in $|z|<1$, and thus if $m=\min _{|z|=1}|h(z)|=$ $\min _{|z|=1}|p(z)|$, then $m \leq|h(z)|$ for $|z| \leq 1$. Now for $\lambda$ with $|\lambda|<1$, we have

$$
|\lambda m|<m \leq|h(z)|
$$

where $|z|=1$.

It follows by Rouche's Theorem that the polynomial $h(z)-\lambda m$ has no zero in $|z|<1$. Hence the polynomial $G(z)=z^{s}(h(z)-\lambda m)=p(z)-\lambda m z^{s}$, has no zero in $|z|<1$ except $s$-fold zeros at the origin. Therefore the polynomial

$$
H(z)=z^{n+s} \overline{G(1 / \bar{z})}=Q(z)-\bar{\lambda} m z^{n},
$$

will have all its zeros in $|z| \leq 1$ with $s$-fold zeros at the origin, where $Q(z)=z^{n+s} \overline{p(1 / \bar{z})}$. Also $|G(z)|=|H(z)|$ for $|z|=1$.

Now, using a similar argument as used in the proof of Lemma 2.7 (inequality (2.4)), for the polynomials $H(z)$ and $G(z)$, we have

$$
\left|z D_{\alpha} G(z)+\beta \frac{(n+s)(|\alpha|-1)}{2} G(z)\right| \leq\left|z D_{\alpha} H(z)+\beta \frac{(n+s)(|\alpha|-1)}{2} H(z)\right|,
$$

where $|\alpha| \geq 1,|\beta| \leq 1$ and $|z|=1$. Substituting for $G(z)$ and $H(z)$ in the above inequality, we conclude that for every $\alpha, \beta$, with $|\alpha| \geq 1,|\beta| \leq 1$ and $|z|=1$,

$$
\begin{aligned}
& \left|z D_{\alpha} p(z)-\lambda((n-s) z+s \alpha) m z^{s}+\beta \frac{(n+s)(|\alpha|-1)}{2}\left(p(z)-\lambda m z^{s}\right)\right| \\
\leq & \left|z D_{\alpha} Q(z)-\bar{\lambda} \alpha n m z^{n}+\beta \frac{(n+s)(|\alpha|-1)}{2}\left(Q(z)-\bar{\lambda} m z^{n}\right)\right|,
\end{aligned}
$$

i.e.,

$$
\begin{aligned}
& \left|z D_{\alpha} p(z)+\beta \frac{(n+s)(|\alpha|-1)}{2} p(z)-\lambda\left((n-s) z+s \alpha+\beta \frac{(n+s)(|\alpha|-1)}{2}\right) m z^{s}\right| \\
\leq & \left|z D_{\alpha} Q(z)+\beta \frac{(n+s)(|\alpha|-1)}{2} Q(z)-\bar{\lambda}\left(n \alpha+\beta \frac{(n+s)(|\alpha|-1)}{2}\right) m z^{n}\right| .
\end{aligned}
$$


Since all the zeros of $Q(z)$ lie in $|z| \leq 1$ with $s$-fold zeros at the origin, and $|p(z)|=$ $|Q(z)|$ for $|z|=1$, therefore by applying Theorem 1.1 to $Q(z)$, we have

$$
\begin{aligned}
\left|z D_{\alpha} Q(z)+\beta \frac{(n+s)(|\alpha|-1)}{2} Q(z)\right| & \geq\left|n \alpha+\beta \frac{(n+s)(|\alpha|-1)}{2}\right| \min _{|z|=1}|Q(z)| \\
& =\left|n \alpha+\beta \frac{(n+s)(|\alpha|-1)}{2}\right| m .
\end{aligned}
$$

Then for an appropriate choice of the argument of $\lambda$, we have

$$
\begin{aligned}
& \left|z D_{\alpha} Q(z)+\beta \frac{(n+s)(|\alpha|-1)}{2} Q(z)-\bar{\lambda}\left(n \alpha+\beta \frac{(n+s)(|\alpha|-1)}{2}\right) m z^{n}\right| \\
= & \left|z D_{\alpha} Q(z)+\beta \frac{(n+s)(|\alpha|-1)}{2} Q(z)\right|-|\lambda|\left|n \alpha+\beta \frac{(n+s)(|\alpha|-1)}{2}\right| m,
\end{aligned}
$$

where $|z|=1$.

Then combining the right hand sides of (3.4) and (3.5), we can rewrite (3.4) as

$$
\begin{aligned}
& \left|z D_{\alpha} p(z)+\beta \frac{(n+s)(|\alpha|-1)}{2} p(z)\right|-|\lambda|\left|(n-s) z+s \alpha+\beta \frac{(n+s)(|\alpha|-1)}{2}\right| m \\
\leq & \left|z D_{\alpha} Q(z)+\beta \frac{(n+s)(|\alpha|-1)}{2} Q(z)\right|-|\lambda|\left|n \alpha+\beta \frac{(n+s)(|\alpha|-1)}{2}\right| m
\end{aligned}
$$

where $|z|=1$.

Equivalently

$$
\begin{aligned}
& \left|z D_{\alpha} p(z)+\beta \frac{(n+s)(|\alpha|-1)}{2} p(z)\right| \\
\leq & \left|z D_{\alpha} Q(z)+\beta \frac{(n+s)(|\alpha|-1)}{2} Q(z)\right|-|\lambda|\left\{\left|n \alpha+\beta \frac{(n+s)(|\alpha|-1)}{2}\right|\right. \\
& \left.-\left|(n-s) z+s \alpha+\beta \frac{(n+s)(|\alpha|-1)}{2}\right|\right\} m .
\end{aligned}
$$

As $|\lambda| \rightarrow 1$ we have

$$
\begin{aligned}
& \left|z D_{\alpha} p(z)+\beta \frac{(n+s)(|\alpha|-1)}{2} p(z)\right| \\
\leq & \left|z D_{\alpha} Q(z)+\beta \frac{(n+s)(|\alpha|-1)}{2} Q(z)\right|-\left\{\left|n \alpha+\beta \frac{(n+s)(|\alpha|-1)}{2}\right|\right. \\
& \left.-\left|(n-s) z+s \alpha+\beta \frac{|\alpha|-1}{2}\right|\right\} m .
\end{aligned}
$$


It implies for every real or complex number $\beta$ with $|\beta| \leq 1$ and $|z|=1$,

$$
\begin{aligned}
& 2\left|z D_{\alpha} p(z)+\beta \frac{(n+s)(|\alpha|-1)}{2} p(z)\right| \\
\leq & \left|z D_{\alpha} p(z)+\beta \frac{(n+s)(|\alpha|-1)}{2} p(z)\right|+\left|z D_{\alpha} Q(z)+\beta \frac{(n+s)(|\alpha|-1)}{2} Q(z)\right| \\
& -\left\{\left|n \alpha+\beta \frac{(n+s)(|\alpha|-1)}{2}\right|-\left|(n-s) z+s \alpha+\beta \frac{(n+s)(|\alpha|-1)}{2}\right|\right\} m .
\end{aligned}
$$

This in conjunction with Lemma 2.7 gives for $|\beta| \leq 1$ and $|z|=1$,

$$
\begin{aligned}
& 2\left|z D_{\alpha} p(z)+\beta \frac{(n+s)(|\alpha|-1)}{2} p(z)\right| \\
\leq & \left\{\left|n \alpha+\beta \frac{(n+s)(|\alpha|-1)}{2}\right|+\left|(n-s) z+s \alpha+\beta \frac{(n+s)(|\alpha|-1)}{2}\right|\right\} \max _{|z|=1}|p(z)| \\
& -\left\{\left|n \alpha+\beta \frac{(n+s)(|\alpha|-1)}{2}\right|-\left|(n-s) z+s \alpha+\beta \frac{(n+s)(|\alpha|-1)}{2}\right|\right\} \min _{|z|=1}|p(z)| .
\end{aligned}
$$

The proof is complete.

Acknowledgements. The authors wish to thank the referee, for the careful reading of the paper and for the helpful suggestions and comments.

\section{REFERENCES}

[1] A. Aziz and Q. M. Dawood, Inequalities for a polynomial and its derivative, J. Approx. Theory 54 (1998), 306-313.

[2] A. Aziz and W. M. Shah, Some inequalities for the polar derivative of a polynomial, Proc. Indian Acad. Sci. 107 (1997), 263-270.

[3] A. Aziz and W. M. Shah, Inequalities for a polynomial and its derivative, Math. Ineq. Appl. 7 (2004), 379-391.

[4] S. Bernstein, Sur la limitation des derivees des polnomes, C. R. Acad. Sci. Paris. 190 (1930), $338-341$.

[5] M. Bidkham, M. Shakeri and M. E. Gordji, Inequalities for the polar derivative of a polynomial, J. Ineq. Appl. 1155 (2009), 1-9.

[6] T. N. Chan and M. A. Malik, On Erdös-Lax theorem, Proc. Indian. Acad. Sci. 92 (1983), 191-193.

[7] K. K. Dewan and S. Hans, Generalization of certain well-known polynomial inequalities, J. Math. Anal. Appl. 363 (2010), 38-41.

[8] K. K. Dewan and A. Mir, Inequalities for the polar derivative of a polynomial, J. Interdisciplinary Math. 10 (2007), 525-531.

[9] K. K. Dewan, N. Singh and A. Mir, Extension of some polynomial inequalities to the polar derivative, J. Math. Anal. Appl. 352 (2009), 807-815.

[10] P. D. Lax, Proof of a conjecture of P. Erdos on the derivative of a polynomial, Bull. Amer. Math. Soc. 50 (1944), 509-513. 
[11] A. Liman, R. N. Mohapatra and W. M. Shah, Inequalities for the polar derivative of a polynomial, Complex Anal. Oper. Theory. 6 (2012), 1199-1209.

[12] M. Marden, Geometry of Polynomials: Mathematical Surveys, American Mathematical Society, Providence, USA, 1966.

[13] Q. I. Rahman and G. Schmeisser, Analytic Theory of Polynomials, Oxford University Press, New York, 2002.

[14] A. Zireh, On the maximum modulus of a polynomial and its polar derivative, J. Inequal. Appl. 111 (2011), 1-9.

${ }^{1}$ Department of Mathematics, Shahrood University of Technology, Shahrood, Iran.

E-mail address: azireh@shahroodut.ac.ir

${ }^{2}$ Department of Mathematics,

University of Semnan, Semnan, Iran

E-mail address: mdbidkham@gmail.com 